\title{
Keynote address: The human and health significance of parent-infant contact
}

\author{
JOHN H. KENNELL, M.D
}

Cleveland, Ohio

The theme of the 1987 AOA Research Conference is "Human Contact and Health." That is an appealing and exciting topic-appealing as an opportunity to learn some of the things that are not known about the effects of human contact, and exciting as we consider its importance and how much more we can discover about the connections between human contact and health through research.

Much of the research I will discuss is of studies that my long-time colleague $\mathrm{Dr}$. Marshall Klaus and I conducted together. ${ }^{1}$ Our interest in parent-infant contact and our research questions come from our experiences caring for premature and sick newborn infants and their parents. Therefore, much of this presentation will be centered on events around childbirth and early infancy. Some of our studies looked at the effects of providing extra mother-infant contact which can be considered the opposite of the separation that had been a prominent feature of the care of healthy and sick newborns in U.S. hospitals. Separation is a common feature in our U.S. cultureand was institutionalized in the separation of mothers and babies after delivery, and family members from the mother during labor and delivery - until relatively recently. As I proceed with questions and observations, you may appreciate that human contact is an important component in many of the issues to be discussed, for example, breast-feeding.

All of us who are fortunate to work with pediatric patients and in the maternity hospital where the family is born have a unique opportunity to relate parental and infant behavior to biology and the underlying physiology. It is my hope that this research conference will stimulate us to take advantage of this unique opportunity so that we can be of more help to parents, infants, and children.

Dr. Klaus and I were launched into our studies of parent-infant bonding by our heavy clinical experiences with premature and sick newborns and their parents. In working with them we saw the great difficulties faced by parents starting with their young infants after a separation, manifest at times by tragic outcomes such as child abuse and nonorganic failure to thrive. Studies of the effects of separation of parents from their premature infants led us to question the effects of the 8- to 12-hour separation of mothers from their healthy full-term infants, which was the standard practice in the United States 15 years ago. Along with this separation, these babies were cared for almost completely by nurses and were brought to mothers every 4 hours for a 20 - or 30 -minute feeding. At this time there was an extremely low incidence of breast-feeding.

Animal studies were a helpful guide to us in planning studies at first, but the greatest impetus to our research and to a fresh view of birthing practices, parentinfant interaction, and child rearing came from anthropology, crosscultural observations, and an evolutionary perspective.

Anthropologic data now suggest that the human species has not significantly changed physiologically or genetically for one to two million years. During this time period, we have had the same skull and bone structure, the same number of chromosomes, and perhaps many of the same behavior patterns that humans have today. During most of this period we humans lived as hunters and gatherers. Thus our physiologic processes and genetic makeup were probably adapted to the life of hunting and gathering during this long time period (Fig. 1). ${ }^{2}$ The small square in the lower right hand corner indicates the approximately 10,000 years that we humans have had the agricultural mode of existence. The 200-year span of our present industrialized society is represented by an almost invisible dot, a period too short for evolutionary adjust- ments to occur. This long history of evolutionary adaptation during which our biology, molded by the environment, slowly evolved practices related to childbirth and the raising of young children can provide the conceptual framework for productive research. The behavior of hunters and gatherers is a source of many interesting ideas but doesn't necessarily tell us what to do about parents and infants today. It may be most profitable to use observations of the behavior of hunters and gatherers, as well as those noted almost universally in agricultural societies, to generate questions about human physiologic processes that can then be tested in industrial societies. Let's use this approach and consider some factors that influence early parent-infant communication.

\section{Doula: Support during labor}

Dr. Klaus and I studied who was present with the mother during labor using the Murdock and White Anthropological Sample of 186 geographically, linguistically, and historically representative nonindustrialized societies. All the major known cultural types in the world are represented by the selection of a single one in each of the 186 differentiated areas, ensuring maximal independence of the societies from each other. We examined the ethnographic material available

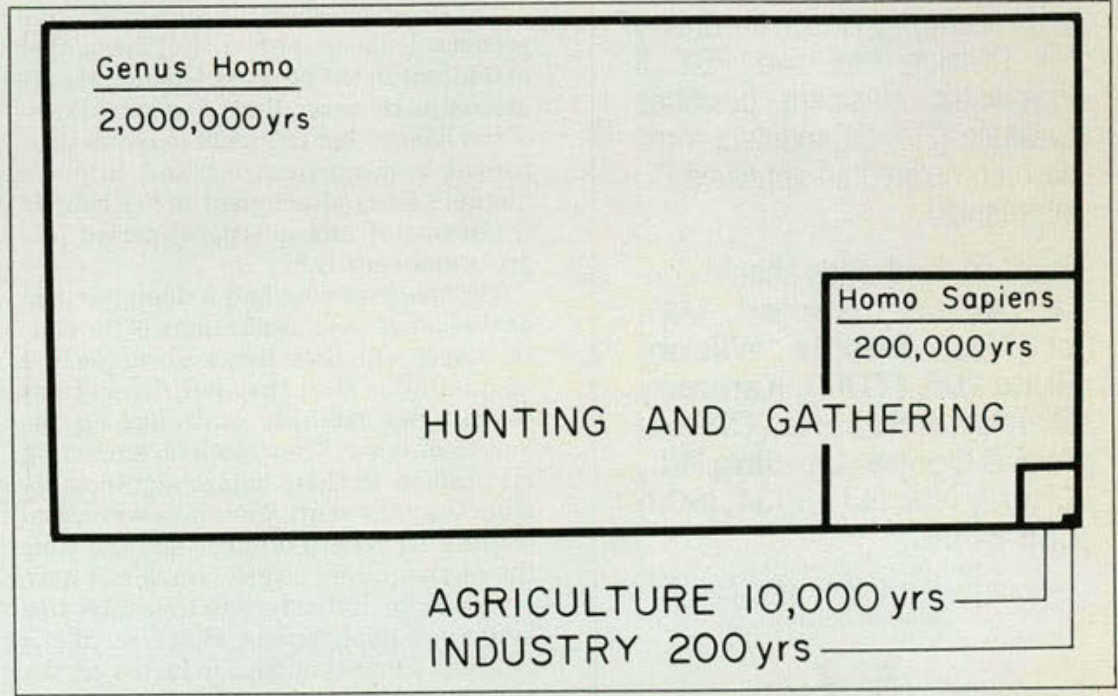

Fig. 1. Schematic diagram illustrating the relative time humans have spent as industrial and agricultural societies compared with their long duration as hunters and gatherers. (From Lozoff, B.: Developmental Medicine and Child Neurology 25:595, 1983). 
in 128 societies. In 127 of the 128 societies, a woman was present with the mother-tobe throughout the labor, and in only one society did the mother labor alone.

Before childbirth moved from the home to the hospital in the twentieth century, it was also the practice in industrialized nations for family members to support the mother actively in labor, often with the assistance of a trained or untrained midwife. Although more fathers, relatives, and friends have been allowed into labor and delivery rooms in the past 20 year $(27$ percent of fathers were in the delivery room in 1973; 79 percent in 1983), a considerable number of mothers still undergo labor and delivery in some hospitals without the presence of family members or close friends. Even though fathers are allowed in many hospitals, they frequently are unsure, ill at ease, often in the way, and not able to provide active support.

Guided by observations of the clinical usefulness of continuous social support during labor, we systematically studied its clinical effect on maternal and infant morbidity in an obstetric setting in Guatemala in which mothers routinely go through labor alone. A total of 417 healthy primigravidous women were enrolled using a randomized design. ${ }^{3}$ The experimental group received support from a woman who was not a midwife, physician, or nurse. We have called this

\section{PSYCHIATRIC RESIDENTS}

\begin{abstract}
The University of Medicine and Dentistry of New Jersey's School of Osteopathic Medicine at Kennedy Memorial Hospital's Cherry Hill Division has two PGY II Psychiatric Resident positions available $7 / 1 / 88$. Candidate must have completed an approved DO internship.
\end{abstract}

\section{Qualified candidates should con- tact: William Ranieri, D.O. or Mrs. Bonnie Wilson, Suite 206, (TDO), Kennedy Memorial Hospital, Chapel Ave. \& Cooper Landing Rd., Cherry Hill, NJ 08034, (609) 665-6080.}

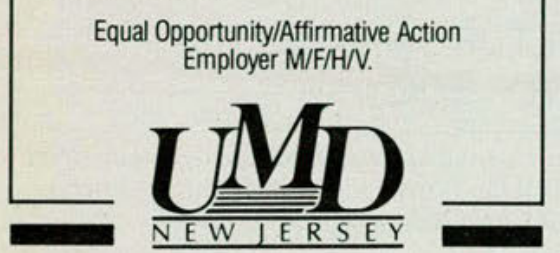

supportive companion a doula, a term introduced by Dana Raphael. The doula provided social support continuously throughout labor and delivery. The control group of mothers labored alone, which was the routine practice in the hospital in which the study was conducted. This routine was established by physicians and nurses from the United States in the $1950 \mathrm{~s}$. The 168 women receiving support throughout labor had significantly fewer perinatal complications (34 vs. 74 percent). Medication was prescribed significantly more often in the control group (19 vs. 4 percent). The rate of cesarean section was significantly greater in the control group also (19 vs. 7 percent). With a continuous caregiver the length of labor was significantly shorter ( 8 vs. 14 hours). These were all highly significant differences. Interestingly there was also a trend for a smaller percentage of infants to be admitted to the Neonatal Intensive Care Unit from the group of mothers who had a continuous caregiver ( 2.4 vs. 6.8 percent). It should be emphasized that all the mothers had a normal pregnancy and were healthy at the beginning of labor

These observations of the direct effects of continuous social support suggest it is possible that our understanding of the normal birth process might be improved by observing common behaviors in nonindustrialized societies. In her insightful book, "Birth in Four Cultures," Brigitte Jordan pointed out the extraordinary extent to which practitioners buy into their own systems' moral and technical superiority whether in the U.S., Sweden, or in Mexico. 4

She described the delivery system in the Yucatan. The woman is never alone. This is an absolute requirement. A mother is surrounded by people she knows intimately at all times. We know from our own society that women can labor alone and are highly adaptable, but there may be a major cost to women in the present arrangements in some hospitals.

The direct effects of the continuous support of a doula on the health of the mother and infant in the study in Guatemala are dramatic. However, there appear to be indirect effects that influence early motherinfant communication and make a mother's early attachment to her baby in the neonatal and puerperal period progress more easily. ${ }^{5}$

The mothers who had a doula present during labor were awake more of the time they were with their newborns in the first 22.5 minutes after they left the delivery room, if we carefully controlled for the length of labor. They stroked, smiled at, and talked to their babies significantly more than the control mothers when controlling for length of labor and the time the mothers were awake. We do not have evidence to indicate whether this has long-term implications. However, in the original study that we conducted on the effects of early and extended mother-infant contact, there are clues suggesting that the level of involvement between mothers and infants during the early postpartum period might significantly affect later behavior.

Further study will be necessary to determine the long-range impact of continuous support during labor on the relationship of the mother and baby, father and baby, and the mother and father Our findings from the doula research are particularly relevant to the care of mothers where positive family support may not be provided during labor and delivery, and where there may be no cultural preparation for childbirth; for example, young, poor, deprived, unmarried, or minority mothers.

How do we decide who will provide positive support? Is the father of the baby adequately prepared to fill the role of the doula? What about other famiy members? Until these issues have been studied we must be careful about jumping to conclusions. In the United States it is the practice to expect all fathers to serve as the supportive companion during labor. However, this is different than the custom in 127 of 128 representative societies. In those where the father may be permitted near the laboring woman he played a minimal role while an experienced woman managed the delivery. Nonsystematic observations in private and public obstetric units and in a pilot study to examine the behavior of male partners during labor and delivery 6 show that some fathers provide excellent support but other fathers do not appear in the hospital or leave early. Even well-prepared fathers may find the strain too great.

Experiences with the doula supporting the couple, the mother and father, during labor and delivery suggest strongly that the doula's support of the father may enable him to be more supportive toward his wife.

In one hospital where we had research experience with 125 deliveries, in which the father or another family member was present, there was consistent and enthusiastic appreciation for the role of the doula. Further research is needed to determine how a doula can work best with a couple.

Even as research is underway to investigate the effectiveness of the doula in the United States, doulas are beginning to provide support for mothers and for couples. Many have a longer period of association with the mother than in our research. This seems highly desirable and is more like the practices in most nonindustrialized societies where close family members or friends also provide support in the prenatal period and protect, feed, and support the new mother with her baby in the first days, weeks, and months. The doulas in the United States often provide support to the mother in relating to the baby and in establishing breast-feeding during the postpartum days in the hospital and the first difficult days of adjustment at home.

In summary, the doula is a low-cost intervention that may be a simple way to reduce the length of labor, cesarean sections, and a number of perinatal prob- 
lems. However, there is a real hazard that the supportive companion will be considered less important than medical interventions. Because it does not fit the medical model of care, it may not be provided as it should be, for every mother in all hospitals. A constant companion during labor should be just as strong an imperative or requirement in industrialized nations as it is for the Mayan Indians of the Yucatan

\section{Communication with the fetus}

Visualization by ultrasound has made it possible to observe the response of the fetus to visual and auditory stimuli. It is clear that the fetus is able to see, hear, taste, and respond to touch in the uterus. By some complicated process the fetus can be affected by its mother's emotional experiences. Thus the mother and her baby are engaged in a dialogue long before the baby's birth. The activity level and emotional state of the mother affects the cycles of the fetus so that he or she is familiar with the tempo of her life at the time of birth. When a bright light is flashed on the mother's abdomen, the fetus will blink after one second delay. In contrast, there is an immediate blink in response to a loud sound. The opportunity for parents to visualize the purposeful movements and behaviors of the fetus such as smiling, yawning, stretching, and grasping has resulted in a revolutionary effect on the timing of attachment. Seeing the small details of the fetus (sometimes even before the usual time of quickening) appears to accelerate the normal attachment process.

Shortly after birth a full-term infant will choose to hear his own mother's voice in preference to other women's voices. There is no similar preference for the father's voice until after the newborn period. If a woman reads the same story, "The Cat in the Hat," to her fetus twice a day in the last 6 weeks of her pregnancy, he or she will choose to hear that story after birth in preference to another story, "The King, the Mice and the Cheese," which in turn will be selected by a newborn who has heard that story repeatedly in utero. Recent research suggests it is the rhythm of the story rather than the words that are recognized and chosen by the infant.

\section{Species specific behavior}

Some specific kinds of maternal behavior such as nesting, retrieving, grooming, and exploring, are seen in nonhuman mammalian mothers immediately before, during, and after delivery. These have evolved to meet the needs of the young in the animal's usual environment. For example, the domestic cat, whether in the United States, China, Russia, or England, behaves in a characteristic way at the time of the birth of her kittens. Towards the end of her pregnancy, she is less active and agile than usual, doing markedly less jumping and climbing. She finds a warm dark place, preferably with a soft surface, in which to give birth. Through labor and delivery, she spends increasingly more time licking her genital region. After delivery, she continues licking, but with the newborn kitten as her object. The placenta is usually promptly eaten. After the birth of the last kitten, the mother lies down, encircling her kittens, and rests with them for about 12 hours. Nursing, which may begin as early as 30 minutes after birth, appears to be stimulated by her licking. For the first two days, she stays with her litter at the birth site. ${ }^{7}$

We wondered if there was a similar species specific behavior in the human mother. A stimulus to explore this subject was the unusual behavior we observed when we first invited mothers of premature infants into the nursery in the first days and weeks of life. On the first visit, mothers walked round the incubator rather warily. When shown how to put their hands into the incubator, they would only briefly and hesitantly poke the baby's extremities with their fingertips. They were frightened and often unable to take an active role. ${ }^{8}$ After observing this many times, we decided to examine in detail the behavior of mothers of full-term and premature infants during the first minutes and hours of their initial interactions.

An orderly and predictable pattern of behavior was observed when each of the mothers of the full-term infants first saw her infant. Commencing hesitantly with fingertip contact on the extremities, within 4 to 5 minutes she began caressing the trunk with her palm, simultaneously showing progressively heightened excitement, which continued for several minutes. Her activity then often diminished, sometimes to such a degree that she fell asleep with the infant at her side. Figure 2 summarizes the changes made by the mothers of full-term infants during three time periods, in fingertip and palm contact on either the extremities or trunk. When behavior in the first and third periods is compared, there is a decrease in fingertip contact from 52 to 26 percent, an increase in palm contact from 28 to 62 percent, a decrease in touching the infant's extremities from 38 to 22 percent, and an increase in touching the infant's trunk from 24 to 49 percent. 9

Mothers of premature infants studied on their first three contacts (over a period of from 1 to 17 days), exhibited an attenuated sequence of the behavior observed in the mothers of full-term infants during the first contact. Figure 3 shows the amount of maternal palm and fingertip activity recorded with premature infants during the first three hospital visits. The progression from fingertip to palm contact did not occur over the time span of the first three contacts in mothers of premature infants. Instead, fingertip contact increased in the second and third contacts. ${ }^{9}$

At present, it is not possible to state whether there are specific patterns of behavior after delivery in the human or how one would go about determining whether or not the observed behavior is characteristic. However, it is interesting to see recurring patterns in several different delivery situations, for example, the movement from fingertip to palm contact and touching, first of the extremities and then of the trunk, in both hospital and home deliveries in the United States. Similar

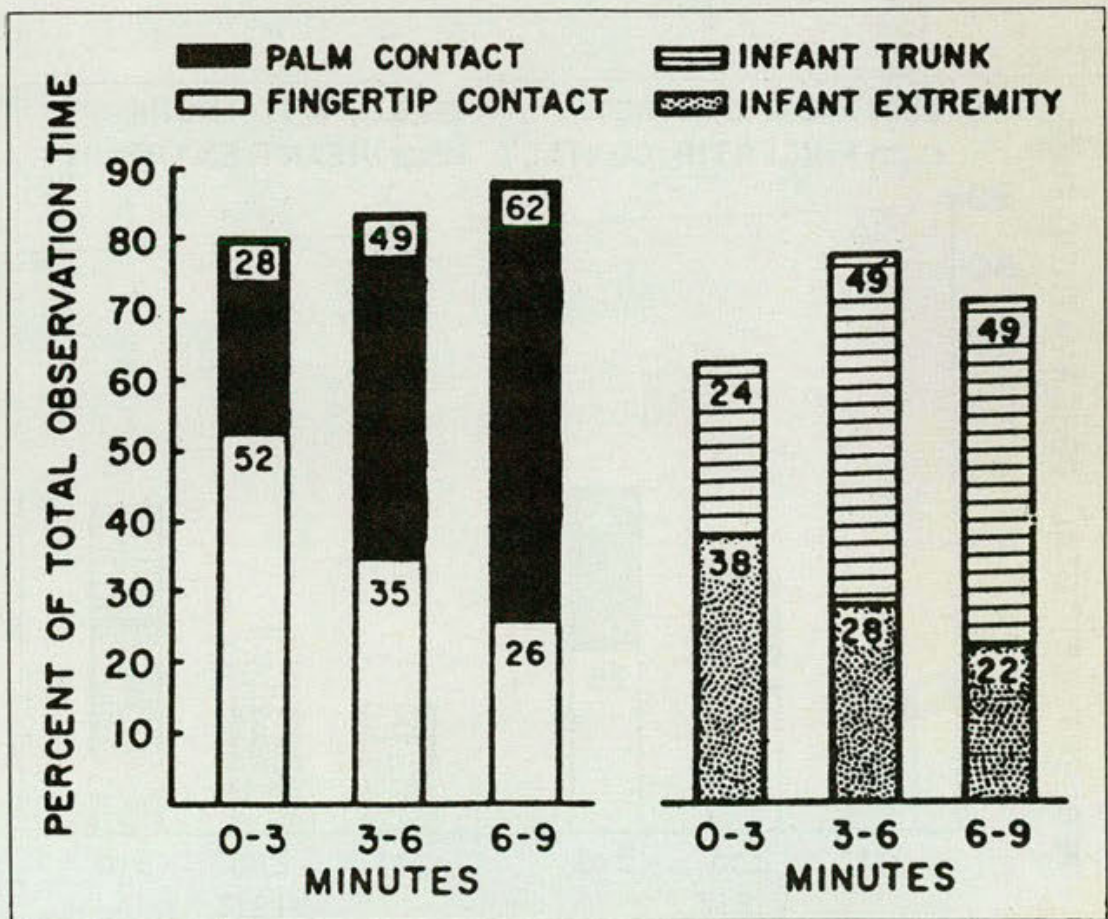

Fig. 2. Palm and fingertip contact on the trunk and extremities at the first postnatal contact in mothers of full-term infants. (From Klaus, M.H., Kennell, J.H., Plumb, N., Zuehlke, S.: Pediatrics 46:187, 1970. Reproduced by permission of Pediatrics). 
patterns have been noted at the first contact of unmarried students and young siblings with undressed newborn infants.

\section{Bonding or biology of attachment}

If one looks at Murdock and White's standard anthropologic sample of 186 representative nonindustrial societies, 183 societies, or all but three, expected mothers and babies to nest together for days or weeks after delivery, a rooming-in equivalent, and virtually none permitted the degree of separation which has been routine in many maternity hospitals in this century. In the past 15 years more than 17 separate studies have focused on whether additional time for close contact of the mother and infant in the first minutes, hours, and days of life alters the quality of attachment. ${ }^{1}$

In three studies, the extra time for mother-infant contact was added not only during the first 3 hours after delivery, but also during the next 3 days. In one of these studies, measurements were made at 1 month and at 1 and 2 years. At 1 month, the mothers in the group that had extra contact showed significantly more affectionate behavior toward their infants. They stood close and watched them more during the physical examination, touched and soothed them more when they cried, engaged in more eye-to-eye contact and fondling with them during feeding, and were more reluctant to leave them with someone else. At 1 year, the two groups of mothers were again significantly different, with extra contact mothers spending more time assisting and soothing their infants during stressful office visits. At 2 years, the linguistic behavior of the two groups was compared.
Extra contact mothers used significantly more words per proposition and nearly twice as many questions, but fewer content words and imperatives than did the controls.

In 13 studies the additional mother-infant contact occurred only during the first hour of life. In 10 of these studies significant differences in the behavior of the mothers were noted. In 6 of 9 studies the duration of breast-feeding was significantly increased by permitting the mother additional time with her infant in the first hour.

In one example of a study with no mother-infant contact in the first 8 hours, O'Connor and associates, ${ }^{10}$ in research on 301 patients, noted significant differences in parenting disorders-child abuse, neglect, abandonment, and nonorganic failure to thrive during the first year of life. They found 10 parenting disorders in the control group and two in the group of mothers who were given extended contact.

The effects of increased mother-infant contact in these 17 studies may be due in part to a recapitulation of what was previously normal human maternal behavior. Increasing mother-infant contact any time during the first 3 postpartum days may trip an innate system in women that has been present in our genetic make-up for centuries. The effects of increasing mother-infant contact may in part make up for the marked deprivation that is a part of present-day routines in modern hospitals.

\section{Mother-infant interaction}

Keeping the mother and baby together soon after birth is likely to initiate and

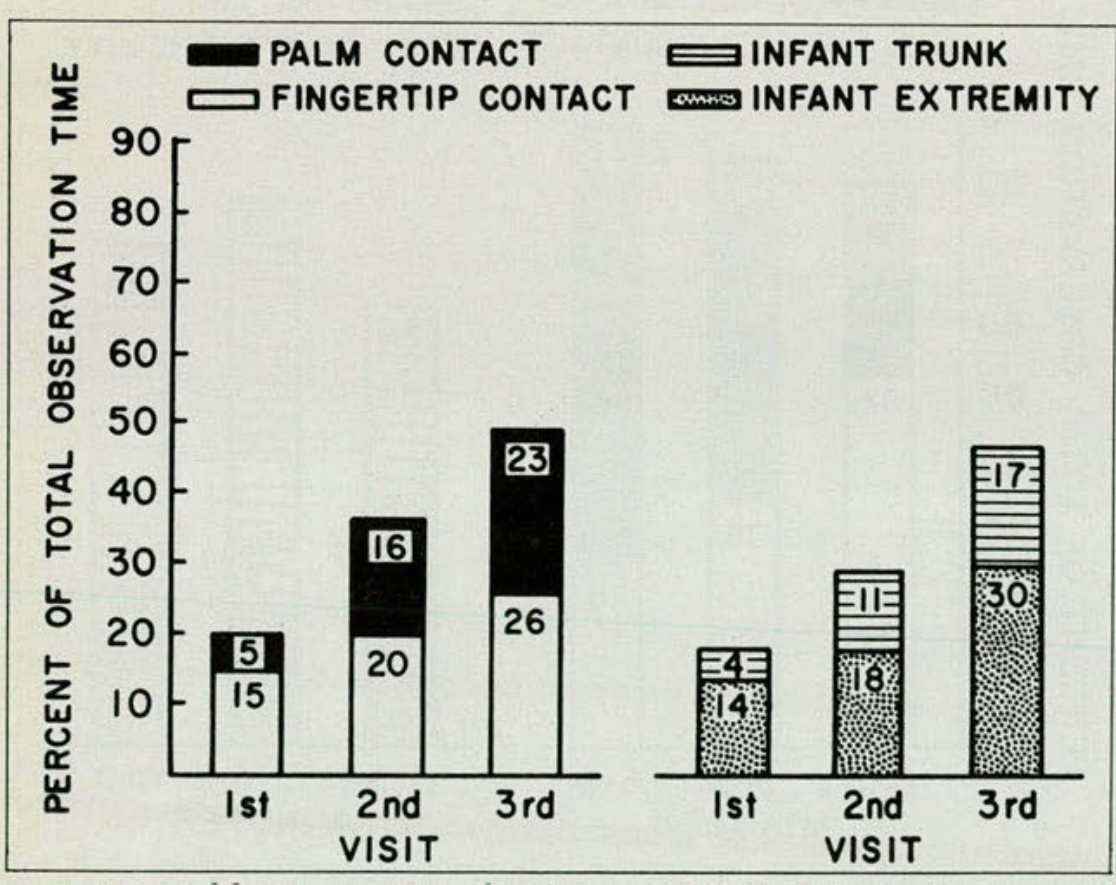

Fig. 3. Palm and fingertip contact on the trunk and extremities at the first three postnatal visits in 9 mothers of premature infants. (From Klaus, M.H., Kennell, J.H., Plumb, N., Zuehlke, S.: Pediatrics 46:187, 1970. Reproduced by permission of Pediatrics). enhance the operation of known sensory, hormonal, physiologic, immunologic, and behavioral mechanisms that probably attach the parent to the infant. Figure 4 shows a few of the multiple reciprocal factors that are in play during the first 3 days a mother is with her infant.

We hypothesize that a cascade of interactions between the mother and baby occurs during this period, locking them together and ensuring the further development of attachment.

Studies have shown that human mothers can identify their infants by smell alone 6 hours after birth after a single 30 minute exposure to their babies. This ability persists to 48 hours. Husbands can recognize their wives' odor but fathers didn't recognize their baby's odor. Eidelman and colleagues ${ }^{11}$ recently reported that mothers who had more than 10 minutes exposure to their newborn infants had 90 percent successful identification of their infants' shirt by smell. With more than 1 hour of exposure success was 100 percent.

Infants can identify their mothers by odor as shown by MacFarlane ${ }^{12}$ and other investigators.

Formby ${ }^{13}$ showed that mothers recognize and select the cry of their own baby from a tape recording of 5 cry sequences. Before 48 hours from delivery, 12 of 23 mothers recognized their own infant's cry. After 48 hours, all 8 mothers recognized their own infant's cry.

Mothers in multi-bedded wards reported 15 of 26 waking episodes were for their own babies on the first and third night. After that 22 of 23 wakings were for their own infants.

\section{Support and mother-infant interaction}

Anisfeld and Lipper ${ }^{14}$ showed that a group of mothers who had contact with their infants in the first hour after delivery showed much more affectionate interaction with their newborn infant 48 hours after delivery when compared to mothers who experienced routine separation. In a careful analysis of their data, they discovered that the effects of this early contact were strikingly increased with the group of mothers who had a low level of social support, that is, two or more of the following: single, on public assistance, had not graduated from high school, and the father or other family member was not present in the delivery room. In contrast, mothers with the same lack of social support showed the lowest level of affectionate interaction with their newborns when they experienced the routine separation after delivery.

In the 183 out of 186 representative nonindustrial societies that expected mothers and babies to nest together for days or weeks after delivery, the early rooming-in was usually followed by extensive mother-infant contact and prolonged and frequent breast-feeding during the early months. Does that protected and supported, extended, postpartum period together in the first weeks, the period after discharge from the hospital,

continued on page $642 / 125$ 


\section{non-insulin-dependent}

\section{Diabetes and hypertension}

Because non-insulin-dependent diabetes mellitus is frequently accompanied by hypertension, it is important to select an oral hypoglycemic agent that rarely causes water retention. In contrast to chlorpropamide, MiCRONASE, when administered alone, may produce a mild diuresis. ${ }^{1}$

All sulfonylureas, including MiCRONASE, can cause severe hypoglycemia. Proper patient selection, dosage, and instructions are important.

No other oral antidiabetic agent fits the realities of life better than

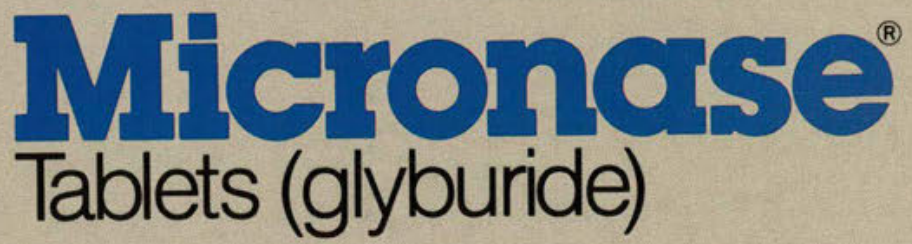

Usual starting dosage $-2.5 \mathrm{mg}$ to $5 \mathrm{mg}$ once a day

When diet alone fails...MICRONASE

Please see adjacent page for brief summary of prescribing information.

'Moses AM, Howanitz J, Miller M: Diuretic action of three sulfonylurea drugs. Ann Intern Med 1973;78:541-544.

\section{Upjohn}



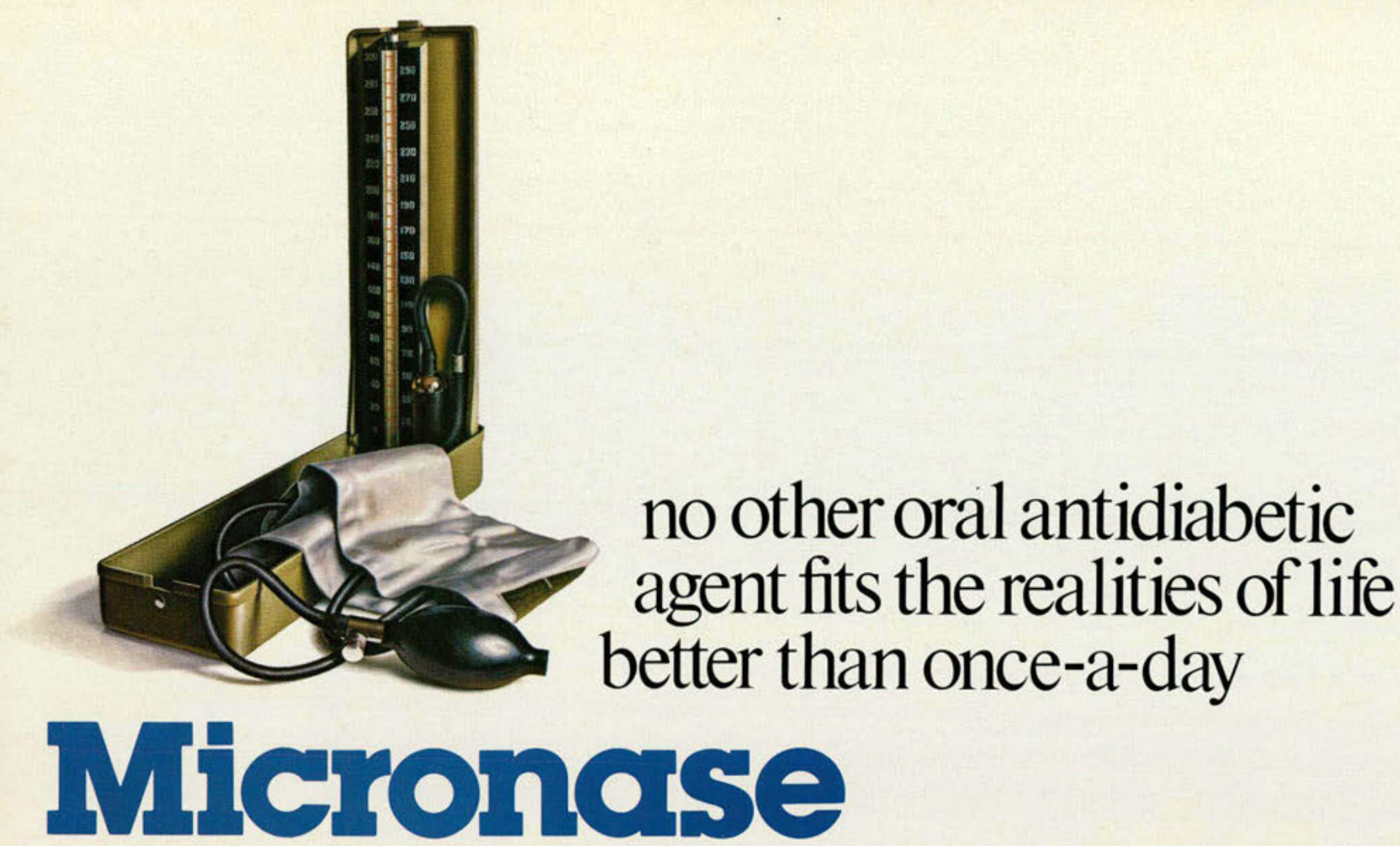

Some patients, particularly those receiving more than 10 mg daily, may have a more satisfactory response with twice-a-day dosage.

Micronase Tablets (glyburide)

CONTRAINOICATIONS: MICRONASE Tablets are contraindicated in patients with: 1. Known hypersensitivity or allergy to the drug. 2. Diabetic ketoacidosis, with or without coma. This condition should be treated with insulin. 3. Type I diabetes mellitus, as sole therapy.

SPECIAL WARNING ON INCREASED RISK OF CARDIOVASCULAR MORTALITY: The administration of oral hypoglycemic drugs has been reported to be associated with increased cardiovascular mortality as compared to treatment with diet alone or diet plus insulin. This warning is based on the study conducted by compared to treatment with diet alone or diet plus insulin. This warning is based on the study conducted by
the University Group Diabetes Program (UGDP), a long-term prospective clinical trial designed to evaluate the University Group Diabetes Program (UGDP), a long-term prospective clinical trial designed to evaluate
the effectiveness of glucose-lowering drugs in preventing or-delaying vascular complications in patients with noninsulin-dependent diabetes. The study involved 823 patients who were randomly assigned to one of four treatment groups (Diabetes, 19 [Suppl 2):747-830, 1970).

UGDP reported that patients treated for 5 to 8 years with diet plus a fixed dose of tolbutamide ( 1.5 grams per day) had a rate of cardiovascular mortality approximately $2 \frac{1}{2}$ times that of patients treated with diet alone. A significant increase in total mortality was not observed, but the use of tolbutamide was discontinued based on the increase in cardiovascular mortality, thus limiting the opportunity for the study to show an based on the increase in cardiovascular mortality, thus limiting the opportunity for the study to show an
increase in overall mortality. Despite controversy regarding the interpretation of these results, the findings increase in overall mortality. Despite controversy regarding the interpretation of these results, the findings potential risks and advantages of MICRONASE and of alternative modes of therapy.

Although only one drug in the sulfonylurea class (tolbutamide) was included in this study, it is prudent from a safety standpoint to consider that this warning may apply to other oral hypoglycemic drugs in this class. in view of their close similarities in mode of action and chemical structure.

PRECAUTIONS: General-Hypoglycemia: All sulfonylureas are capable of producing severe hypoglycemia Proper patient selection and dosage and instructions are important to avoid hypoglycemic episodes. Renal o hepatic insufficiency may increase the risk of serious hypoglycemic reactions. Elderly, debilitated or malnourished patients, and those with adrenal or pituitary insufficiency, are particularly susceptible to the hypoglycemic action of glucose-lowering drugs. Hypoglycemia may be difficult to recognize in the elderly and in people who are taking beta-adrenergic blocking drugs. Hypoglycemia is more likely to occur when caloric intake wis deficient, ater severe or prolonged exercise, When alcohnis is mosted or when more than one glucose patients exposed to stres such as fever, trauma, infection or surgery, a loss of control may occur. It may then be necessary to discontinue MICRONASE and administer insulin. Adequate adjustment of dose and adherence to diet should be assessed before classifying a patient as a secondary failure. Information for Patients: Patients should be informed of the potential risks and advantages of MICRONASE and of alternative modes of therapy. They also should be informed about the importance of adherence to dietary instructions, of a regular exercise program, and of regular testing of urine and/or blood glucose. The risks of hypoglycemia, its symptoms and treatment, and conditions that predispose to its development should be explained to patients and responsible family members. Primary and secondary failure should also be explained. Laboratory Tests: Response to MICRONASE Tablets should be monitored by frequent urine glucose tests and periodic blood glucose tests. Measurement of glycosylated hemoglobin levels may be helpful in some patients. Drug Interactions: The hypoglycemic action of sulfonylureas may be potentiated by certain drugs including nonsteroidal antiinflammatory agents and other drugs that are highly protein bound, salicylates, sulfonamides, chloramphenicol, probenecid, coumarins, monoamine oxidase inhibitors, and beta adrenergic blocking agents. Certain drugs tend to produce hyperglycemia and may lead to loss of control These drugs include the thiazides and other diuretics, corticosteroids, phenothiazines, thyroid products, estrogens, oral contraceptives, phenytoin, nicotinic acid, sympathomimetics, calcium channel blocking drugs, and isoniazid. A potential interaction between oral miconazole and oral hypoglycemic agents leading to severe hypoglycemia has been reported. Carcinogenesis, Mutagenesis, and Impairment of Fertility: Studies in rats at doses up to $300 \mathrm{mg} / \mathrm{kg} / \mathrm{day}$ for 18 months showed no carcinogenic effects. Glyburide is nonmutagenic when studied in the Salmonella microsome test (Ames test) and in the DNA damage/alkaline elution assay. Pregnancy: Teratogenic Effects Pregnancy Category B. Reproduction studies in rats and rabbits have revealed no evidence of impaired fertlity or harm to the fetus due to glyburide. There are no adequate and well controlled studies in pregnant women. This drug should be used during pregnancy only if clearly needed. Insulin should be used during pregnancy to maintain blood glucose as close to normal as possible. Nonteratogenic Effects: Prolonged severe hypoglycemia ( 4 to 10 days) has been reported in neonates born to mothers who were receiving a sulfonylurea drug at the time of delivery. MICRONASE should be discontinued at least two weeks before the expected delivery date. Nursing Mothers: Some sulfonylurea drugs are known to be excreted in human milk. Insulin therapy should be considered. Pediatric Use: Safety and effectiveness in children have not been Insulin therapy
established.

ADVERSE REACTIONS: Hypoglycemia: See Precautions and Overdosage sections. Gastrointestinal Reactions: Cholestatic jaundice may occur rarely: MICRONASE Tablets should be discontinued if this occurs. Gastrointestinal disturbances, e.g. nausea, epigastric fullness, and heartburn, are the most common reactions, having occurred in $1.8 \%$ of treated patients during clinical trials. They tend to be dose-related and may disappear when dosage is reduced. Liver function abnormalities, including isolated transaminase elevations, have been reported. Dermatologic Reactions: Allergic skin reactions, eg pruritus, erythema urticaria, and morbilliform or maculopapular eruptions, occurred in $1.5 \%$ of treated patients during clinical trials. These may be transient and may disappear despite continued use of MICRONASE; if skin reactions trials. These may be transient and may disappear despite continued use of MICRONASE; if skin reactions
persist, the drug should be discontinued. Porphyria cutanea tarda and photosensitivity reactions have been reported with sulfonylureas. Hematologic Reactions: Leukopenia, agranulocytosis, thrombocytopenia, hemolytic anemia, aplastic anemia, and pancytopenia have been reported with sulfonylureas. Metabolic Reactions: Hepatic porphyria and disulfiram-like reactions have been reported with sulfonylureas; however. hepatic porphyria has not been reported with MICRONASE and disulfiram-like reactions have been reported very rarely. Cases of hyponatremia have been reported with glyburide and all other sulfonylureas, most often in patients who are on other medications or have medical conditions known to cause hyponatremia or increase release of antidiuretic hormone. (SIADH) secretion has been reported with certain other sulfonylureas, and it has been suggested that these sultonylureas may augment the peripheral (antidiuretic) action of ADH and it has been suggested that the increase release of $\mathrm{ADH}$.

OVERDOSAGE: Overdosage of sulfonylureas, including MICRONASE Tablets, can produce hypoglycemia. If hypoglycemic coma is diagnosed or suspected, the patient should be given a rapid intravenous injection of concentrated $(50 \%)$ glucose solution. This should be followed by a continuous infusion of a more dilute (10\%) glucose solution at a rate which will maintain the blood glucose at a level above $100 \mathrm{mg} / \mathrm{dL}$. Patients should be closely monitored for a minimum of 24 to 48 hours, since hypoglycemia may recur after apparent clinical recovery.

Caution Federal law prohibits dispensing without prescription. Store at controlled room temperature $15^{\circ}-30^{\circ} \mathrm{C}\left(59^{\circ}-86^{\circ} \mathrm{F}\right)$. Dispensed in well closed containers with safety closures. Keep container tightly closed. For additional product information see your Upjohn representative

\section{Upjohn}

THE UPJOHN COMPANY, Kalamazoo, MI 49001, USA 
have significance for our society?

Carrying the baby on the mother's body Most travelers are amazed to note that they almost never hear a baby cry when they visit a nonindustrialized country. Was the human infant meant to cry except when in pain? Is crying due to a need for warmth, contact, and stimulation? The practice of our ancestors, the hunters and gatherers, for hundreds of thousands of years of carrying the baby on the mother's body and sleeping with the baby at night is still carried out in developing countries, which have a settled agricultural existence.

For women in foraging societies, close mother-infant contact with the baby kept in the vertical or semi-vertical position is a common practice in the first months of life. Our associates have recently explored the possibility that this body position might have physiologic benefits for the infant. $\mathrm{TCPO}_{2}$ measurements (partial pressure of blood oxygen measured with a skin electrode) were assessed in normal healthy premature infants while they were awake, in both the supine and partially upright position. ${ }^{15}$ In 12 infants with a postconceptional age of 34 to 36 weeks, there was a $7 \mathrm{~mm}$. increase in $\mathrm{PO}_{2}$ in the semiupright position. In 11 infants from 36 to 38 weeks, there was a slightly smaller but significant increase in $\mathrm{PO}_{2}$, the amount of oxygen in the blood, when the infants were moved from the supine to the upright position. Previously it had been noted in infants that $\mathrm{PO}_{2}$ during sleep is higher when the infant is on the abdomen than in the supine position. ${ }^{16}$ As a consequence, routine care of prematures and full-terms has been changed to keep infants prone rather than supine. There is now additional evidence of less crying, more sleep and better gastric emptying in the prone position.

\section{Mother-infant communication}

Carrying the baby on the parent's body raises the question, how is the human infant built to communicate with his mother? Distally or proximally? In general, mothers in Western Societies use the baby's scream as a cue to communication. Has our society taught mothers to use only auditory and visual rather than tactile communication? In many cultures, every young mother is expected to be able to carry her baby on her body all day and detect the small changes in breathing and body movements that precede urination and defecation, so that by one week she is able to carry her baby with no soiling of her clothes. She is considered a poor mother if she makes a misjudgment. For Eskimo mothers, a miscalculation can be disastrous because of wind-chill factors that result in freezing of the skin with exposure of less than 30 seconds. Mothers and fathers in most industrialized nations seldom learn how to pick up tactile signals from their babies and to achieve this state of fine-tuned responsiveness to the baby's needs. When the mother is breast-feeding and the infant is carried on her body, there is no delay when the baby becomes hungry. By means of infrared photography John Lind showed the rapid effect of infant's cry on the mother's lactating breast. Thoman ${ }^{17}$ in careful observations in the home observed that an infant would stop crying promptly if the mother intervened within the first one and a half minutes. However, if she did not tend to the baby's crying within 90 seconds, the crying would be greatly prolonged.

In Montreal, Canada, three investigators encouraged one group of mothers of young infants to carry the infant in a carrier or in her arms at least three extra hours a day. ${ }^{18}$ When this group of babies was compared with a group managed in the usual way without any extra carrying, there was 44 percent less crying and one-third less fussing, three weeks later. This was most evident in the evening when North American babies traditionally cry and fuss the most. Would carrying the baby on the mother's body

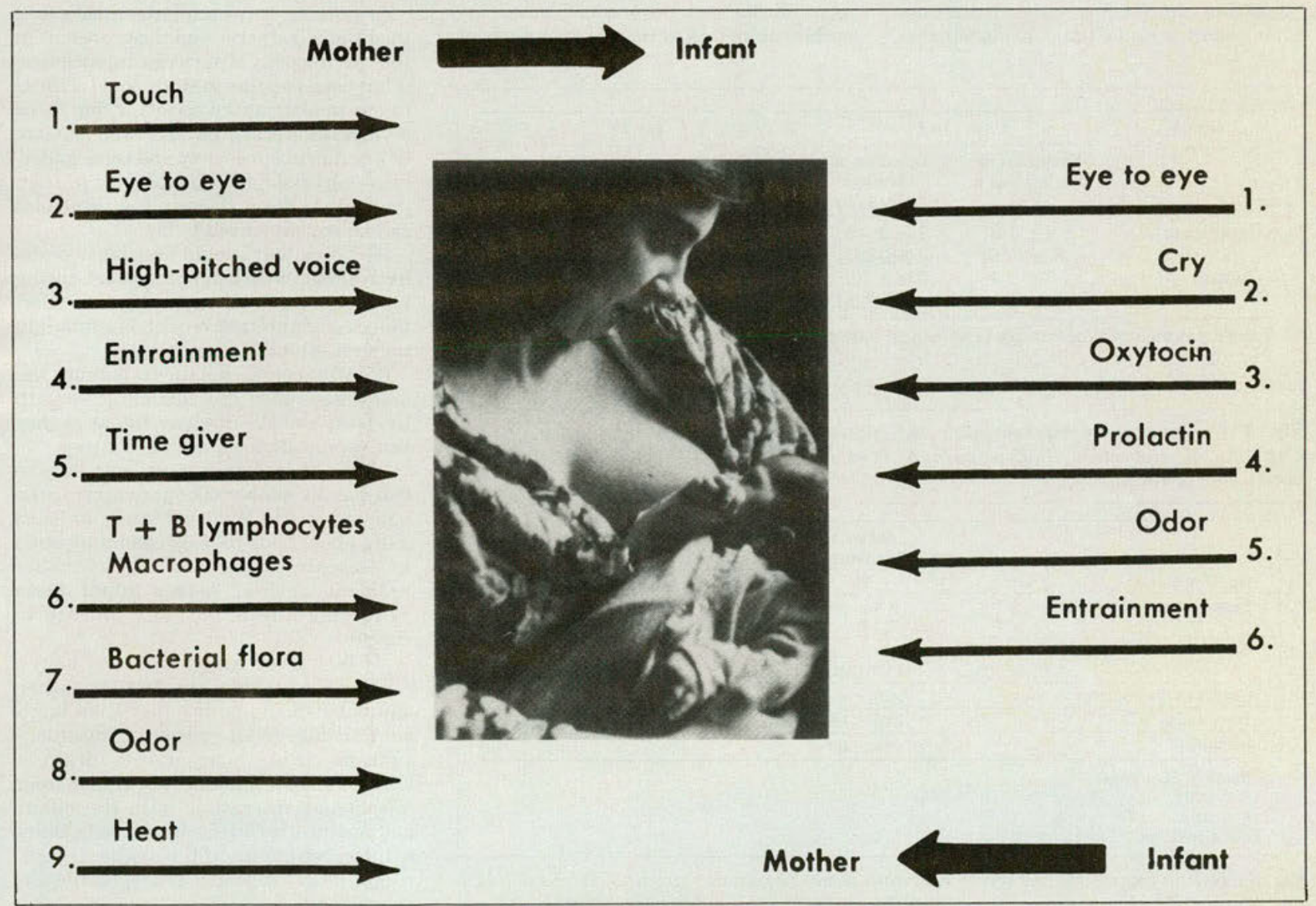

Fig. 4. Mother-to-infant and infant-to-mother interactions that can occur simultaneously in the first days of life. (Reproduced by permission from Klaus, M. and Kennell, J. Parent-Infant Bonding, ed. 2, C.V. Mosby Co., 1982). 
prevent colic? Sudden infant death syndrome? Reduce the incidence of non-

Ways of holding and feeding an infant that have been maintained over hundreds of thousands of years, may prove to have physiologic and adaptive advantages.

\section{Breast-feeding}

The adults and children in the Mayan Indian Village of Santa María Cauque in meticulously by Leonardo Mata and Juan Urrutia for a decade and a half. Almost every mother in that village was able to breast-feed successfully for a mean period of two years. Even mothers with conditions such as mastitis, acute respiratory infections, or Shigella or Salmonella enteritis did not stop breast-feeding. These women had no watches or clocks to time the intervals between feedings and put no soap or ointments on their nipples. They fed the baby whenever the baby wanted to suckle and as often and as long as the baby wished. They slept with their infant so nursing continued every few minutes through the night. It was this frequent nursing that appeared to assure an abundant milk supply and provide maximum contraceptive effect.

Observations of women and their babies in hunting-gathering and agricultural societies reveal that they nurse 30-40 times in 24 hours and each nursing is for a short period. Studies exploring the organic failure to thrive? the Guatemalan highlands were studied

effect of increasing the feeding of nursing mothers have produced information that provides a new understanding of several neonatal physiologic processes and strongly supports keeping the mother and infant together to permit frequent nursing.

In a series of studies of human infants deCarvalho and coworkers ${ }^{19}$ showed that mothers who were encouraged to nurse frequently in the first two weeks compared to mothers breast-feeding on a 3 - to 4-hour schedule ( 9.9 vs. 7.3 feedings per 24 hours) produced significantly more milk ( 725 vs. $502 \mathrm{ml} . / 24$ hours) and their babies gained more weight (Fig. 5). Increasing the length of feedings had no effect on milk production. In a second study mothers who nursed their infants more than 8 times per 24 hours in the first three days of life had significantly lower serum bilirubin levels ( $6.5 \mathrm{vs} .9 .3 \mathrm{mg} . / \mathrm{dl}$.) (Fig. 6).20

Studies by several different investigators suggest that increasing the feeding frequency probably decreases the serum bilirubin by increasing gut motility which increases the fecal excretion of bilirubin, thus decreasing its intestinal absorption. Barbero in unpublished observations noted in 15 neonates increased large intestinal contractions from pressure recordings when the lips of the newborns were rubbed (a labiocolic reflex). Fitting with this argument, Rosta and coworkers ${ }^{21}$ noted hyperbilirubinemia in infants with delayed

\begin{tabular}{|ccccccc}
\hline \multirow{2}{*}{ Group } & \multicolumn{2}{c}{ Days 1 to 14} & & \multicolumn{2}{c}{ Day 15 } \\
\cline { 2 - 3 } \cline { 5 - 6 } & $\begin{array}{c}\text { Frequency of } \\
\text { Feedings/24 } \mathrm{h}\end{array}$ & $\begin{array}{c}\text { Duration of } \\
\text { Feedings } \\
\text { (min/24 }\end{array}$ & & $\begin{array}{c}\text { Milk Intake } \\
(\mathrm{mL} / 24 \mathrm{~h})\end{array}$ & $\begin{array}{c}\text { Weight Gain from } \\
\text { Birth }(\mathrm{g})\end{array}$ \\
\hline Experimental & $9.9 \pm 1.9 \ddagger$ & $134 \pm 48$ & & $725 \pm 171 \ddagger$ & $561 \pm 286 \dagger$ \\
& $(6.8-13.6)$ & $(66-223)$ & & $(368-1,045)$ & $(216-847)$ \\
Control & $7.3 \pm 1.4$ & $138 \pm 39$ & & $502 \pm 185$ & $347 \pm 331$ \\
& $(5.4-10.4)$ & $(74-225)$ & & $(294-902)$ & $(-555-858)$ \\
\hline
\end{tabular}

*Values are means $\pm \mathrm{SD}$; range is shown in parentheses.

$+P<.02$.

$\ddagger P<.001$

Fig. 5. Comparison of experimental and control groups on days 1 to 15. (From De Carvalho, M., Robertson, S., Friedman, A., et al: Pediatrics 72:307, 1983. Reproduced by permission of Pediatrics).

\begin{tabular}{|c|c|c|}
\hline & $\begin{array}{c}\text { Group 1, } \\
\leq 8 \text { Feedings } / 24 \mathrm{hr}\end{array}$ & $\begin{array}{c}\text { Group 2, } \\
>8 \text { Feedings } / 24 \mathrm{hr}\end{array}$ \\
\hline No. of subjects & 29 & 26 \\
\hline Feeding frequency $/ 24 \mathrm{hr}$ & $\begin{array}{l}6.8 \pm 0.8^{\circ} \\
(5.3-8)\end{array}$ & $\begin{array}{l}10.1 \pm 1.6 \dagger \\
(8.3-15.3)\end{array}$ \\
\hline Serum bilirubin, $\mathrm{mg} / \mathrm{dL}$ & $\begin{array}{l}9.3 \pm 3.5 \\
(3.5 \cdot 15.5)\end{array}$ & $\begin{array}{l}6.5 \pm 4.0 \ddagger \\
(1.5 \cdot 12.5)\end{array}$ \\
\hline Length of feeding, min & $14.3 \pm 4$ & $13.3 \pm 3.7 \S$ \\
\hline Weight loss, $\mathrm{g}$ & $219 \pm 86$ & $216 \pm 59 \S$ \\
\hline Hematocrit, \% & $54 \pm 6.5$ & $55 \pm 7.8 \S$ \\
\hline $\begin{array}{l}- \text { Mean } \pm S D \text { (range). } \\
\uparrow P<.0001 . \\
\ddagger P<.01 . \\
\S \text { Not significant. }\end{array}$ & & \\
\hline
\end{tabular}

Fig. 6. Feeding frequency and serum bilirubin levels. (From de Carvalho, M., Klaus, M., and Merkatz, R.: Frequency of breast-feeding and serum bilirubin concentration, American Journal of Diseases of Children, 136:737, 1982, copyright 1982, American Medical Association. Reproduced by permission.) passage of meconium

Other studies that support increasing the nursing frequency reveal decreased breast pain and nipple soreness at higher feeding frequencies as well as increased success in lactation.

Evidence from another arena gives further support for increasing feeding frequencies. Comparative measurements of the fat and protein constituents of milk from many mammals suggests that the human pattern of infant care in the past was nearly continuous feeding and carrying (Fig. 7). Infant animals in species adapted for long separations (feeding every 4-12 hours) receive milk with a high protein and fat concentration and reduced water, while infant mammals who are carried or follow their mothers and feed almost continuously receive milk with a low protein and fat concentration. Human milk is low in fat and extremely low in protein. From the anthropologic history of infant care, Lozoff ${ }^{22}$ suggests that human babies were probably carried and nursed frequently for more than 99 percent of our species' existence. To ensure survival, human babies were carried close to the body of the mother, not only to be fed but also to be kept warm.

We believe there is now sufficient evidence to question whether any human mother should be limited to 4 -hour feedings if she is interested in breast-feeding successfully.

In summary, by noting the practices of the hunter-gatherer and those present in the vast majority of agricultural societies, it has been possible to study some of these in our industrialized society. From these studies some practices have been found to be of value in our society and some underlying physiologic principles have been uncovered. From these a few principles can be recommended today.

(1) No mother should ever labor or deliver alone. With human support during labor a mother will be more active, affectionate and interactive with her infant in the first minutes after delivery.

(2) We should explain to parents the many ways they can communicate with the fetus and the newborn infant so they can appreciate his amazing abilities.

(3) We should encourage carrying the baby on the mother's body or carrying the baby as in the Montreal study to learn more about body-to-body communication to decrease crying, and perhaps other problems such as sudden infant death syndrome and nonorganic failure to thrive.

(4) Whenever the mother and baby's condition warrants, the mother, father and baby should be together from birth on. This may reduce parenting disorders such as child abuse and nonorganic failure to thrive, enhance attachment and affectionate interaction with the infant and increase breast-feeding. The benefits will be even greater if the mother is poor, single, poorly educated, teenaged and unsupported.

(5) The baby should be kept with the parents throughout the first days and the 


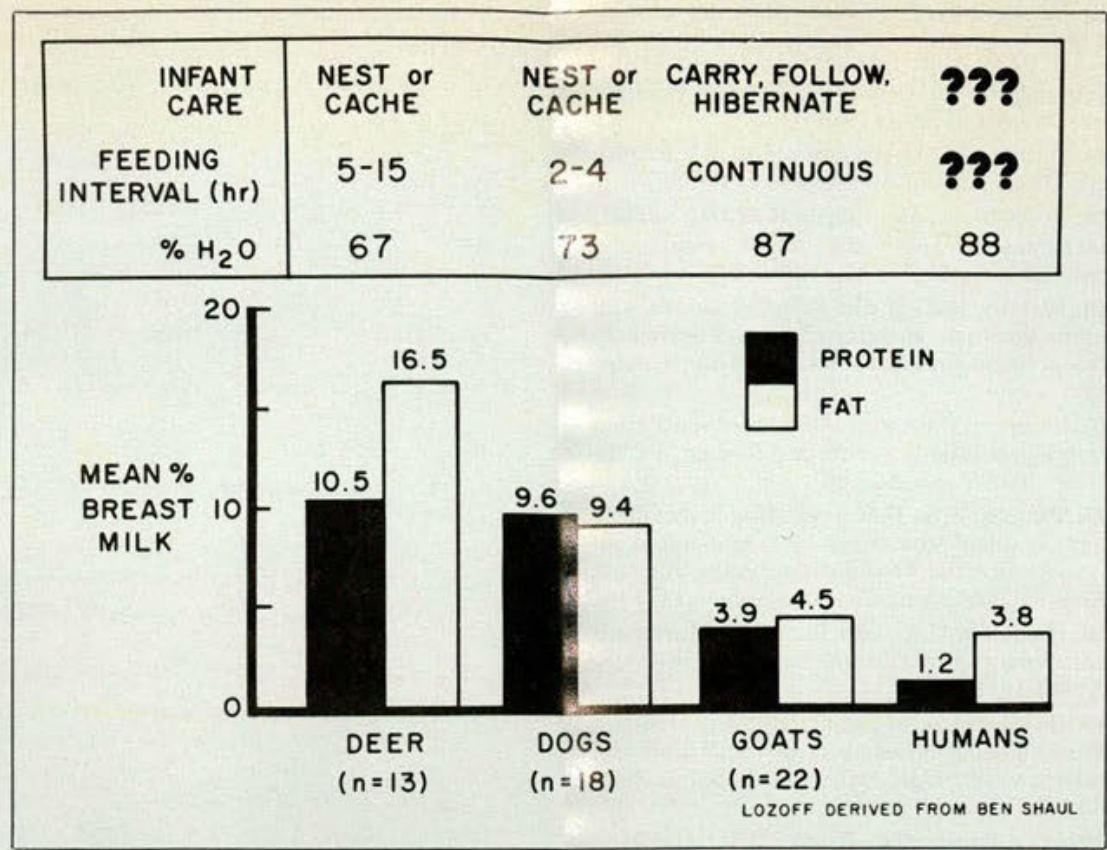

Fig. 7. Relationship of feeding interval to fat and protein concentration and percentage of water. The differences in fat and protein concentrations between the groups are significant. $n=$ Number of species studied. (Modified from Lozoff, B., Brittenham, G.M., Trause, M.A., et al: J. Pediatrics 91:1, 1977. Reproduced by permission of the C.V. Mosby Co.).

central nurseries should be closed because: (a) there will be less risk of infection; (b) the mother and infant will have an optimal opportunity to interact; (c) the mother will be familiar with the baby's early signals, needs, and schedule, so she will be better prepared to meet his needs when she takes him home; (d) with mother and baby together the baby can nurse frequently, feed whenever he is hungry, obtain a larger volume of milk sooner, gain weight faster, and have an increased probability of successful breast-feeding with all its nutritional, immunologic, and psychologic benefits; (e) the baby will cry less; (f) mothers will detect symptoms and signs of problems earlier; and (g) with more frequent breast-feedings babies will have lower levels of bilirubin and less need for laboratory studies and phototherapy.

There is a wide range of adaptability present in human mothers, fathers, and infants. Studies suggest that there may be considerable cost due to modern adaptations in the care of parents and newborn infants. The studies reported here have yielded valuable biobehavioral findings but do not necessarily suggest what should be recommended behavior in our industrialized society. The striking similarity of some of the behaviors across many cultures other than our own suggests they may have biologic survival value and may more often be linked to generalizable, physiologic or biologic principles.

Explorations of other biological systems associated with the behavior of hunters and gatherers as well as the universal practices in agricultural societies may provide a valuable guide to creative and novel studies of touch and human contact that could further benefit mothers, fathers, and infants throughout the world. Compared to the large number of studies of other sensory systems, touch is a neglected area. As indicated in this presentation, touch is a frequent and a key element in behavior during labor, delivery, and infancy. It may have importance at times of high emotional impact throughout life, for example, at times of separation and reunion, in lovemaking, and at times of stress. Studies of social support, such as the doula during labor, are showing health benefits with many medical and social conditions. There is even evidence of improvement in immune system function with social suport. How much of the effects of what is called social support is due to touch and human contact? There is a need and a great opportunity for research. 
1. Klaus, M.H., and Kennell, J.H.: Parent-infant bonding, Ed. 2. C. V. Mosby Co., St. Louis, 1982

2. Lozoff, B.: Birth and 'bonding' in non-industrial societies. Dev Med Child Neurol 25:595-600, Oct 83

3. Klaus, M.H., et al.: Effects of social support during parturition on maternal and infant mordibity. Brit Med J 293:585, 1986

4. Jordan, B.: Birth in four cultures. Eden Press Women's Publications, St. Albans, New Jersey, 1980

5. Sosa, R., et al.: The effect of a supportive companion on perinatal problems, length of labor, and mother-infant interaction. N Eng $\mathrm{J}$ Med 303:597-600, 11 Sep 80

6. Nagashima, K., et al.: Fathers during labor. Do we expect too much? Pediatric Res 21:62A: Apr 87

7. Schneirla, T.C., Rosenblatt, J. S., and Tobach E.: Maternal behavior in mammals, edited by H. R. Rhengold. John Wiley \& Sons, Inc., New York, 1963

8. Barnett, C.R., et al.: Neonatal separation. The maternal side of interactional deprivation. Pediatrics 45:197-205, Feb 70

9. Klaus, M.H., et al.: Human maternal behavior at the first contact with her young. Pediatrics 46:187-92, Aug 70

10. O'Connor, S., et al.: W.A. Reduced incidence of parenting inadequacy following rooming-in. Pediatrics 66:176-82 Aug 80

11. Eidelman, A., et al.: Mother's recognition of their newborns by olfactory cues. Pediatric Res 21:801A, Apr 87
12. MacFarlane, J.A.: Olfaction in the development of social preferences in the human neonate. In Parent-infant interaction, Ciba Foundation Symposium 33. Elsevier Publishing Co., Amsterdam, 1975

13. Formby, D.: Maternal recognition of infant's cry. Develop Med Child Neurol 9:293, 1967

14. Anisfeld, E., and Lipper, E.: Early contact, social support and mother-infant bonding. Pediatrics 72:79-83 Jul 83

15. Martin, R.J., et al.: Effect of supine and prone positions on arterial oxygen tension in the preterm infant. Pediatrics 63:528-31, Apr 79

16. Shivpuri, C.R., et al.: Decreased ventilation in preterm infants during oral feeding. J Pediatrics 103:285-89, Aug 83

17. Thomas, E.B.: How a rejecting baby affects mother-infant synchrony. In Parent-infant interaction, Ciba Foundation Symposium 33. Elsevier Publishing Co., Amsterdam, 1975

18. Hunziker, U.A., and Barr, R.G.: Increased carrying reduces infant crying. Pediatrics 72:641-48, May 86

19. De Carvalho, M., et al.: Effect of frequent breast-feeding on early milk production and infant weight gain. Pediatrics 72:307-11, Sept 83

20. De Carvalho, M. Klaus, $\mathrm{M}$. katz, R.B.: Frequency of breast-feeding and serum bilirubin concentration. Am J Dis Chil 136:737-8 Aug 82

21. Rosta, J., Makoi, Z., and Kertész, A. Delayed meconium passage and hyperbilirubinemia. Lancet $2: 1138-2,23$ Nov 68

22. Lozoff, B., et al.: The mother-newborn relationship. Limits of adaptability. J Pediat 91:1-12, Jul 77

Dr. Kennell is a professor of pediatrics, Case Western Reserve University School of Medicine, and attending pediatrician, Rainbow Babies and Childrens Hospital, University Hospitals of Cleveland, 2074 Abington Road, Cleveland, Ohio 44106. 